\title{
Paul Ricoeur: o el reconocimiento como experiencia de donación mutua
}

\author{
María Gabriela Rebok*
}

Resumen: En este artículo se aborda la importancia del reconocimiento mutuo en la filosofía socio-política contemporánea, desde la perspectiva de Ricoeur, y se marca la recepción, pero también la diferencia, respecto de las propuestas de Hegel y su actualización en Honneth. Destacamos que el aporte peculiar de Ricoeur consiste en su énfasis, puesto tanto en el lado activo como en el pasivo del reconocimiento, en un camino que atiende sobre todo a las huellas del don y su agradecimiento, como también a la centralidad del recibir. El nervio es una cierta "generosidad del deseo", su carácter festivo-ceremonial y simbólico, que culmina en estadosdepaz y ágape. El reconocimiento así posibilitado en un régimen de sobreabundancia se revela como fundadorderelación.

Palabras clave: Ricoeur, reconocimimiento mutuo, don, paz, ágape.

Abstract: This article gives an approach, from Ricoeur's perspective, to the relevance of the idea of mutual acknowledgment in the socio-political contemporary philosophy. Thus, Hegel's proposal of this concept and the reception as well as the critical actualisation by Axel Honneth are outlined. Further, we explain that the peculiar contribution of Ricoeur consists in the emphasis on the active as well on the passive role of acknowledgment, by means of a way that takes in view all themarks of gift and his connotations of giving thanks, making clear, then, also the central position of receiving. The nerve of this flexible lattice is a certain "generosity of desire", that characterizes its festivity character in a ceremonial and symbolic way, which ends in the state of peace and ágape. So the concept of acknowledgement, as possible in a horizon of abundance and plenty, reveals itself as a founding relationship.

Keywords: Ricoeur, mutual acknowledgment, gift, peace, ágape

*Doctora y Licenciada en Filosofía por la Universidad del Salvador, Buenos Aires. Becaria de la Heinrich-Hertz-Stiftung para estudios de posgrado en la Universidad de Köln (Alemania). Investigadora del CONICET, de la Academia Nacional de Ciencias de Buenos Aires y del Hegel-Archiv de la Ruhr-Universität-Bochum (Alemania). Dirección electrónica: mgrebok@gmail.com. 
Ricoeur retoma el gran Leitmotiv hegeliano a través de la mediación "actualizada" de A. Honneth ${ }^{1}$, quien pertenece a la última generación de la Escuela de Frankfurt. Le reconoce a Hegel el haber superado la hipótesis hobbesiana de la "guerra de todos contra todos" y haberle dado una connotación ética a las relaciones socio-políticas con su propuesta de la "lucha por el reconocimiento". Para ello, es decisivo el concepto hegeliano de laSittlichkeit. Por su parte, acusa la importancia de la contribución de Honneth, quien trata la "lucha por el reconocimiento" de Hegel a nivel de vinculos afectivos, de relaciones jurídicas y de valoraciones sociales. Para Honneth juega un papel destacado la "libertad social".

Según Ricoeur, el camino del reconocimiento recorre tres niveles: describe un arco que va desde la identificación que pretende alcanzar la verdad de una cosa en general, pasando por el reconocimiento de sí como capaz de obrar y ser responsable, hasta el reconocimiento mutuo en la reciprocidad de relaciones con los otros. En cada uno de estos niveles operan los tres registros de la identidad, alteridad y la dialéctica del reconocer/desconocer (reconnaissance/méconnaissance). Basado en la etimología de la lengua francesa (igualmente posible en español), hace su aporte distintivo a una ética de la paz, en la que el dar y el recibir instauran -por encima de la mera reciprocidad y hasta de la nuda justicia - el régimen de la sobreabundancia, propia de la donación. Para ello amplía el horizonte del diálogo, incluyendo a notables antropólogos que investigaron el papel social que cumple el intercambio de dones. Ahora los interlocutores son Marcel Mauss, y las más actuales

1 Es preciso, sin embargo, señalar que -a pesar de algunas semejanzas, inclusive formales- con Honneth, la propuesta de Ricoeur tiene rasgos propios. Honneth recurre a la psicología social de George Herbert Mead como puente entre el planteo hegeliano y el suyo, con fuerte apoyatura también en los recursos empíricos de las ciencias sociales; Ricoeur, por su parte, transita la mediación de su propia antropología filosófica (Segundo Estudio). Asimismo, el recorrido de Ricoeur no culmina en la afirmación de la "libertad social" (Honneth), sino en el ágape, como plenificación del vínculo. Lo empírico, recepcionado por Ricoeur a través del diálogo con antropólogos culturales, historiadores, sociólogos, opera, a mi juicio, como anclaje para una experiencia y concepción no utópicas de la lógica del don, capaz de anticipar el ágape. 
versiones de la problemática en Marcel Hénaff, Mark RoginAnspach y la historiadora NathalieZemon-Davis. A ellos se añaden los sociólogos LucBoltanski y Laurent Thévenot, con quienes discute Ricoeur lo atinente a la justicia y a los estados de paz.

Al titular su obra "camino" o "recorrido", Ricoeur alude a un proceder intermedio entre la teoría y la "rapsodia de ideas", jalonado por "acontecimientos de pensamiento". ${ }^{2}$ Un muy atento intérprete como Jean Greisch señala que en francés "parcours" indica tanto la dirección objetiva del trayecto como el recorrido mismo. En la primera perspectiva se plantea la pregunta acerca de la meta del camino, y la respuesta apunta a la "fenomenología de las fundamentales capacidades humanas". La segunda perspectiva, por su parte, apunta a los descubrimientos que se hacen al andar, aun antes de haber alcanzado la meta. En esta última reconstrucción del camino aparece la memoria responsable del re-conocer como un volver a identificar y la promesa como apertura ética al futuro, ambas cimas del autorreconocimiento. ${ }^{3}$

\section{Punto de partida: el reconocimiento como identificación}

Ricoeur parte del amplio espectro semántico propio de la lengua francesa, una verdadera polisemia, y efectúa un sucinto análisis del lenguaje ordinario. Luego rastrea sus imbricaciones con las huellas dejadas por la historia del pensamiento respecto del reconocimiento. En esta historia detecta un corrimiento en el eje desde la vOz activa del verbo reconocer (reconozco) a la vOZ pasiva (soyreconocido). A su vez, la voz activa señala "el dominio del pensamiento sobre el sentido"; y la voz pasiva, la demanda o solicitud de ser reconocido. ${ }^{4}$ Como "acontecimientos de pensamiento" menciona entre los

${ }^{2}$ Cfrr. Ricouer, PaulCaminos del reconocimiento. Tres estudios, trad. A. Neira, Madrid, Trotta, 2005, pp. 253 s.; Parcours de la reconnaissance. Troisétudes, Paris, Stock, 2004, pp. 357 s. [en adelante $C R$ y $P R$, respectivamente].

${ }^{3}$ Cfr. Greisch,Jean, "VergifteteGeschenke? Die Gabe der Philosophenund die Gegengabe des Anthropologen” (“¿Regalos envenenados? El don de los filósofos y el contradon del antropólogo"), en Hähnel, Martin (Hg.), Memoria und Mímesis. Paul Ricoeur zum 100. Geburtstag (Memoria y mímesis. A Paul Ricoeur en su 100 cumpleaños), Dresden, Text \&Dialog, 2013, p. 64. [La traducción al castellano es nuestra.]

${ }^{4}$ Cfr. CR, p. 254; PR, p. 358. 
modernos a Descartes y a Kant. En ellos predomina, por cierto, la forma activa del reconocer, y equivale a "identificar, aprehender por el pensamiento una unidad de sentido". ${ }^{5}$

Para Descartes, "reconocer" implica distinguir, en el sentido de identificar algo como verdadero o falso. Viene orillado por el desconocer, el "miedo al error", un error al que le es propio desconocerse a sí mismo y que se alimenta de la oscuridad y confusión. ${ }^{6}$ En cambio, para Kant se trata de la relación, conexión o síntesis bajo la condición del tiempo. El juicio que lo efectúa acontece en la intersección de los dos "troncos del conocimiento humano":"Hay dos troncos del conocimiento humano, que proceden acaso de cierta raíz común, pero desconocida para nosotros: la sensibilidad y el entendimiento, por la primera, se nos dan objetos; por la segunda, los pensamos."

Reconocer sigue teniendo la carga cognitiva, sigue siendo conocer, pero ahora desde la perspectiva trascendental, referida a las condiciones de posibilidad a priori de un objeto en general. Una de estas condiciones es precisamente el tiempo, forma de nuestro sentido interno: "El tiempo es la condición formal a priori de todos los fenómenos en general" (\$6a). "El tiempo no es otra cosa que la forma del sentido interno, es decir, la intuición de nosotros mismos y de nuestro estado interior" (\$6b). Lo es inmediatamente para el sentido interno y mediatamente para el externo y así pasa a ser la llave de los secretos del alma.Los cambios sólo son posibles en el tiempo, pero éste es la forma de nuestra sensibilidad.

En la primera edición de la Crítica de la razón pura se mencionan tres síntesis: la "síntesis de la aprehensión en la intuición", la "síntesis de la reproducción en la imaginación" y la "síntesis de la recognición [Rekognition] en el concepto" (p. 55). Leído el tiempo bajo el esquematismo de la relación pone de relieve que "los tres modos del tiempo son la permanencia, la sucesión y la simultaneidad" (A 176, B 219). La categoría de sustancia se evidencia como una relación entre lo que cambia y lo que no cambia, como fundamento, "algo que está siempre (...) que perdure y sea permanente" (A 182, B 225). La

${ }^{5}$ Cfr. Ibidem, p. 49; p. 63.

${ }^{6}$ Cfr. Ibidem, p. 261; pp. 368 s.

${ }^{7}$ Kant, Immanuel, Kritik der reinenVernunft, Akademie Textausgabe, Berlin, W. de Gruyter, 1968 [A 15, B 30]. 
sucesión regulada está a cargo de la segunda Analogía, la de la causalidad: "Todos los cambios suceden según la ley que enlaza la causa y el efecto" (A 189, B 232). Finalmente, la simultaneidad se refleja en los conceptos de "comunidad" o "acción recíproca entre el agente y el paciente". La identificación-reconocimiento de un objeto cualquiera es posible por la "puesta en relación bajo las condiciones del tiempo" (p. 64).

La ruina de la representación (Levinas dixit) y de la autoafección es operada por la convicción contemporánea de la Lebenswelt, el ser-en-elmundo, la corporeidad como experiencia de base.

\section{El reconocimiento de sí}

Aquí el foco se traslada al hombre "actuante y sufriente" para reconocerlo como hombre capaz puesto de relieve por el sí mismo reflexivo o la ipseidad. Se explicitan previamente instancias del "reconocimiento de la responsabilidad" en el mundo griego: el de Homero, el de los trágicos y el de Aristóteles, aunque tal reconocimiento no alcanzara los niveles de la reflexividad moderna.El hombre capaz se identifica con el reconocimiento-atestación que adopta la certeza del "yo puedo". De él se derivan las capacidades del "poder decir", "poder hacer", "poder narrar" y "ser imputable".

Por lo demás, "[1]a problemática del reconocimiento de sí alcanza simultáneamente dos cimas con la memoria y la promesa. La primera mira hacia el pasado; la segunda, hacia el futuro. Pero ambas deben pensarse juntas en el presente vivo del reconocimiento de sí, gracias a algunos rasgos que poseen en común." "Reconocer un recuerdo es reencontrarlo". Supone la latencia de todo lo experimentado y un trabajo contra el olvido y la afirmación de lo propio. Como presente del pasado, la memoria resguarda la identidad-mismidad.La promesa, en cambio, es el presente del futuro. Lucha contra la traición. Constituye la ipseidad e identidad narrativa. Apunta a lo otro. Es la integración de los poderes: del poder decir, actuar, contar o narrar y poder imputarse sus actos. ${ }^{9}$ Asimismo, "[1] as capacidades no se constatan, sino que se atestan." Y en cuanto tales son susceptibles "de apreciación, de

${ }^{8}$ Ricoeur, Paul, CR., p. 119; PR, p. 165.

I'Ibidem, pp. 163-165. 
evaluación". Pero también implican el derecho a la reivindicación fundada en la justicia social. ${ }^{10}$

\section{E1 reconocimiento mutuo}

Como ya se dijo, ocupa un lugar clave la "lucha por el reconocimiento" hegeliana en su calidad de respuesta ética al desafío de Hobbes con la hipótesis del "estado de naturaleza", caracterizado por la "guerra de todos contra todos". Le sigue el análisis de una versión "actualizada" del tema por parte de Axel Honneth, el representante más significativo de la última generación de la Escuela de Frankfurt. Lo que Honneth acentúa es el elemento de filosofía socio-política, en las condiciones actuales de la eticidad (Sittlichkeit), pero se pierde lo especulativo. ${ }^{11}$ Estimo que tanto Honneth como Ricoeur -quien en esto sigue al pensador de Frankfurt- analizan a un Hegel truncado, al limitarse a la función del reconocimiento en la eticidad. Pero, es justo recalcar que, aun dentro de la eticidad, no se considera la importancia paradigmática, subrayada por Hegel, del reconocimiento mutuo entre hermano-hermana, no condicionado por la lucha. ${ }^{12} \mathrm{Si}$ bien es claro que hubo y hay luchas fratricidas, Hegel parece sugerir un comienzo pacífico cuando lo masculino se relaciona con un polo femenino. ${ }^{13}$ No menor es -según Hegella importancia del reconocimiento mutuo alcanzado en la figura del perdón, que señala el traspaso de la moralidad al espiritu absoluto. El perdón (Verzeibung)implica reconciliación (Versöbnung), y la aparición de Dios en la comunidad religiosa. ${ }^{14}$

\footnotetext{
${ }^{10}$ Ibidem, pp. 155 s.; pp. 217 s.

${ }^{11}$ Cfr. Honneth, Axel, KampfumAnerkennung. Zurmoralischen Grammatik sozialerKonflikte(1994), Frankfurt am Main, Suhrkamp, 2012, pp. 7, 275.

${ }^{12}$ Cfr. Hegel, Georg Wilhelm Friedrich, Phänomenologie des Geistes, Johannes Hoffmeister (Hg.), Hamburg, Meiner, 1952, pp. 325 s.

${ }^{13} \mathrm{Cfr}$. Rebok, María Gabriela, La actualidad de la experiencia de lo trágico y el paradigma de Antígona, Buenos Aires, Biblos, 2012, pp. 96-102.

${ }^{14}$ Cfr. Hegel, G. W. F., op.cit., pp. 471 s. En este sentido, es de destacar el ineludible estudio de Siep Ludwig, AnerkennungalsPrinzip der praktischenPbilosophie, Hamburg,Meiner, 2014, por considerar toda la amplitud del horizonte de Hegel ya en la época de Jena.
} 
Sin embargo, por razones de espacio, me concentraré en el aporte específico de Ricoeur, como homenaje al Centenario de su nacimiento (rememorado en 2013). Metodológicamente cabe destacar que Ricoeur ejercita el principio de hospitalidad, que le era tan caro, respecto de los presupuestos detectables tanto en el lenguaje ordinario como en los "acontecimientos de pensamiento" de la historia de la filosofía y los suyos propios. Reitera su mediación en el "conflicto de las interpretaciones".

Su propuesta arranca de una "duda sobre la idea misma de lucha", conservada aun en la "lucha por el reconocimiento", porque sospecha que puede tratarse de una mal disimulada nueva versión de la "conciencia desgraciada", "si los humanos no pudiesen acceder a una experiencia efectiva, aunque simbólica, de reconocimiento mutuo, según el modelo del don ceremonial recíproco." $15 \mathrm{Y}$ da un golpe de timón hacia la "experiencia efectiva" de los "estados de paz" y la excedencia del ágape. Greisch señala con acierto que el intento ricoeuriano es una circulación que relaciona el cuarteto (Geviert) de reciprocidad, mutualidad, estados de paz y ágape. ${ }^{16}$

Ricoeur contribuye con la exploración de la riquísima veta abierta por la significación de reconocimiento equivalente a agradecimiento. Éste se presenta como una alternativa con una gran irradiación que emerge de mediaciones simbólicas irreductibles al orden jurídico o a los intercambios comerciales. Señala un primer obstáculo a su propuesta precisamente en lo que atañe a la mutualidad del reconocimiento. Los modelos de paz que nos son conocidos en Occidente llevan nombres griegos: el eros platónico, la philía aristotélica y el ágape bíblico y posbíblico. Este último parece borrar la mutualidad o el rasgo interpersonal del reconocimiento en la medida en que la generosidad del don no requiere ni espera el contradon o la devolución. Sin embargo, por eso mismo podrá ejercer la función crítica respecto de la lógica de la reciprocidad para abrir el espacio-tiempo "de la mutualidad del don fundada en la idea de reconocimiento simbólico.”17

\footnotetext{
${ }^{15}$ Ricoeur, Paul, CR., p. 161; PR, p. 226. Ricoeur interpreta esta nueva "conciencia desgraciada" como debatiéndose entre sentimientos incurables de victimización y el "mal infinito" de ideales inalcanzables. Cfr. p. 225; p. 318.

${ }^{16} \mathrm{Cfr}$. Greisch, Jean, op.cit., p. 64.

${ }^{17}$ Cfr. Ricoeur Paul, CR., pp. 227 s.; PR, p. 319.
} 


\subsection{El estado de paz bajo el signo de ágape}

La alternativa, propia del ágape, se opone sin duda a la de lucha y también, en cierto modo, a la de justicia. Si bien es verdad que la justicia inhibe la perpetuidad de la venganza, no extingue la lucha, porque suscita nuevos conflictos precisamente por la idea de equivalencia que rige la relación entre crimen y castigo. En una mano sostiene la justicia la balanza, en la otra la espada. Por el contrario, el ágape es sin equivalencia, ignora la comparación y el cálculo. El ágape es la expresión de la "generosidad del deseo". ${ }^{18}$

En cambio, la philia sí incluye la reciprocidad y cierta relación entre la amistad y la justicia. Dicho con palabras de Aristóteles, la amistad se muestra como "lo más necesario para la vida"."Y cuando los hombres son amigos, ninguna necesidad hay de justicia, mientras que aun siendo justos necesitan además de la amistad, y parece que son los justos los que son más capaces de amistad."19 Interpretando a Aristóteles, podemos decir que, si bien se diferencian justicia y amistad, la amistad tiene preeminencia porque - si no se confunde con el amiguismo- incluye la justicia y la supera. Por otro lado, aunque la justicia no conlleva necesariamente la amistad, parece predisponer hacia ella.

A su vez, según Ricoeur, el ágapese distingue del eros platónico, porque su deseo de ascensión no se nutre con el sentimiento de carencia (penía). Por el contrario, hay abundancia de corazón. Lo que cuenta no es la equivalencia, ni el contradon; sustrae la relación con el prójimo y hasta con el enemigo al juicio, a la medida y al cálculo, según el "no juzguéis para no ser juzgados." Habilita la suspensión de la disputa incluso en el juicio. Por su carácter inconmensurable, el ágape queda sin réplica, le es extraña tanto la justificación como la atención a sí. Es una permanencia y un demorarse [que instaura morada]; ignora el arrepentimiento referido al pasado y la espera de una retribución futura. No argumenta al modo de la justicia, pero se deja decir en ejemplos y parábolas.

${ }^{18}$ Cfr. Ibidem, pp. $282 \mathrm{s.}$

19 Aristóteles, Ética a Nicómaco, ed. bilingüe y trad. M. Araujo y J. Marías, Madrid, Instituto de Estudios Políticos 1970, L. VIII, 1155 a 29-32, p. 122. 
El otro se convierte en prójimo, pero éste no es un mero próximo, sino aquel a quien el amor se aproxima, sale a su encuentro. El lenguaje más propio del ágape es la alabanza que expresa el regocijo por el otro visto como más elevado. Un buen ejemplo es el bimno al amor de SanPablo (I, Cor., 13). A su vez, puede adoptar la forma de una celebración en optativo como el Sermón de la montaña: "Bienaventurados los que (...)". Con todo, parece acercarse a la justicia con el mandamiento "Amarás (...)", aunque resulta muy diferente al imperativo y a la coerción moral. También precede a cualquier ley la demanda del amante a la amada: “¡Ámame!” Dicho desde la ternura tiene un rasgo poético vecino al himno y a la bendición. El lenguaje del ágape encuentra su suelo natal en la metáfora que recurre a las analogías con el amor erótico, como en la mística del Cantar de los Cantares. ${ }^{20}$

En suma, mientras el ágape se declara y se proclama; la justicia argumenta al servicio de la disputa en el tribunal, separa la víctima del culpable, evita la venganza, pero no produce paz. La justicia no sólo presenta pruebas en lo penal, sino también en lo comercial, en los derechos y deberes de las personas, distribuye roles y tareas que fijan un lugar social. Es así justicia distributiva, según los lemas: "Dar a cada uno lo suyo" y "Tratar de manera semejante los casos semejantes".

“Se puede tender un puente entre la poética del ágape y la prosa de la justicia, entre el himno y la regla formal?"21, se pregunta Ricoeur. El deseo activado por ágape no lleva la marca de la carencia, sino de la sobreabundancia. Pero opera en el mismo mundo que la justicia, un mundo dominado por la economía de mercado, donde todo tiene un precio, y por códigos sociales que rigen la relación entre el don y el contradon.El insólito personaje de El idiota de Dostoievski, el príncipe Mishkin, tiene un modo conveniente y ajustado de resolver conflictos, que es más que justo. Pero, por lo inhabitual, genera malentendidos. Actuando en el mundo, ágapepierde su pureza de "alma bella" (téngase en cuenta, por ejemplo, los cuestionamientos por las donaciones a la Madre Teresa); la justicia, su seguridad basada en la regla de la equivalencia.

${ }^{20}$ Cfr. Ricoeur, Paul, CR., p. 281.

${ }^{21}$ CR., p. 231; PR, p. 325. 


\subsection{Las paradojas del don y del contradon y la lógica de la reciprocidad}

El Essai sur le don de Marcel Mauss, ${ }^{22}$ presenta al don como una forma arcaica del intercambio mercantil. Para los maoríes de Nueva Zelanda, vale no tanto la obligación de dar, ni la de recibir, sino la de devolver, o sea, el contradon. Esto plantea la paradoja del carácter aparentemente libre y gratuito junto a la coerción e interés de tales prestaciones. Mauss se pregunta qué fuerza hay en la cosa que obliga al donatario a devolver. Toma la respuesta de los mismos aborígenes: la fuerza que vincula en la reciprocidad está en la cosa donada misma. "En las cosas intercambiadas en el potlatch hay una virtud que fuerza a los dones a circular, a ser dados y devueltos". ${ }^{23}$ Esta fuerza se llama "hau".

En la introducción a Essai sur le don, Lévi-Strauss le reprocha a Mauss "mistificar al indígena", asumiendo la interpretación de éste. Sostiene que esta fuerza no es propia de la cosa, sino una necesidad social inconsciente envuelta en las reglas del pensar simbólico. A su vez, Lefort reprocha a Lévi-Strauss su ambición de reducir lo social a un universo calculable de reglas aun contra la intención inmanente de las conductas por las que un hombre confirma a otro en su condición de "otro yo", capaz de actuar como yo actué.Por su parte Mark RoginAnspach, en $\dot{A}$ charge de revanche. Figures élémentaires de la reciprocité, sostiene: "Una relación de reciprocidad no puede reducirse a un intercambio entre dos individuos. Emerge siempre un tercero trascendente, aunque este tercero no sea más que la relación misma que se impone, de pleno derecho, como actor". ${ }^{24}$ Con ello pone de relieve la lógica de la reciprocidad, que -para Ricoeur- es diferente a la de la mutualidad. 25

Tres son las "figuras elementales de la reciprocidad": la venganza, el don y el mercado. Las tres exhiben relaciones sociales circulares. En el caso de la venganza se trata de un círculovicioso: matar al que mató engendra cada vez más asesinos. Aquí el tercero es una violencia sistémica. No es posible el

${ }^{22}$ Mauss, Marcel, Essai sur le don. Forme et raison de l'échangedans les sociétésarchä̈ques, en Sociologie et Anthropologie, PUF, Paris, 1950. [trad. cast. de T. Rubio de MartínRetortillo, Sociología y antropología, Tecnos, Madrid, 1971]

${ }^{23}$ Ibidem, p. 214.

${ }^{24}$ Anspach, Mark Rogin, À charge de revanche. Figures élémentaires de la reciprocité, Seuil, Paris, 2002, p. 5.

${ }^{25}$ Cfr. Ricoeur, Paul, CR, p. 235; PR, p. 331. 
traspaso a un círculovirtuoso sin el sacrificio entendido como acontecimiento de ofrenda que "dona a quien va a donar". En cuanto al mercado, hasta ahora no ha podido superar su reiterada frustración en la justa distribución de bienes.

Pero queda el don en el cual cabe despertar la mutualidad imprescindible para el acontecer del reconocimiento.Entonces se puede afirmar con Jean-Pierre Dupuy, Aux origines de sciencescognitives."Hacer un don en devolución, reconocer la generosidad del primer donante con un gesto correspondiente de reciprocidad, es reconocer la relación de la que el regalo precedente no es más que un vehículo."26

La interacción hace circular el don y asume la relación como apertura al reconocimiento. En este sentido, sostiene Daniela Falcioni que: "El don es en su núcleo un operador simbólico, una fuerza simbólica efectiva, que funda relaciones (...)."27 También Greisch enfatiza que por medio del acontecimiento de la donación se revela el hombre como capaz de una relación y hasta digno de ella. Sin embargo, todo don signa la relación entre donante y donatario más como riesgo y desafío que como un hecho. ${ }^{28}$ Por eso se resiste a ser pensada dentro de un sistema cerrado. En consecuencia, la lógica de la reciprocidad debe ceder el paso a la fenomenología de la mutualidad, es decir, a un reconocimiento "inmanente a las transacciones interpersonales". ${ }^{29}$

\subsection{El intercambio de dones como matriz del reconocimiento mutuo}

En contraste con la reciprocidad que se da en relaciones sistémicas (Lévi-Strauss), Ricoeur rescata la mutualidad de las relaciones entre (importancia del "Zwischen") los actores del intercambio.Con Marcel Hénaff, ${ }^{30}$ enfatiza el carácter ceremonial y no comercial del intercambio y su relación con la índole

26Dupuy Jean-Pierre, Aux origines des sciencescognitives, La Découverte, Paris 1994, p. 59. Citado por Ricoeur Paul, CR, p. 239; PR, p. 336.

${ }^{27}$ Falcioni, Daniela, "Das Band und die Bindungskraft der Gabe" ["El vínculo y la fuerza vincular del don”], en M. Hähnel (ed.), Memoria und Mimesis, p.79. [La traducción del alemán al castellano es nuestra.]

${ }^{28}$ Greisch, Jean, op.cit., p. 54.

29 Ricoeur, Paul, CR, p. 287.

${ }^{30}$ Hénaff, Marcel, Le prix de la vérité: Le don, l'argent, la philosophie, Seuil, Paris, 2002. 
simbólica del reconocimiento. Equivale a algo que "no tiene precio". Empalma también con toda una historia en Occidente en la que se yergue la figura de Sócrates contra los sofistas, por la gratuidad de su enseñanza, y el ciudadano libre de Atenas en cuyo círculo no entran los comerciantes (en su mayoría extranjeros o libertos). La sospecha contra el dinero y la usura atraviesa la Edad Media, se interrumpe con el Renacimiento y la Reforma, pero encuentra nuevos representantes en el siglo XIX con Baudelaire, Flaubert y Marx. ${ }^{31}$

Al designar como "ceremonial" el intercambio del reconocimiento simbólico, Hénaff se opone, por un lado, a la interpretación moralizante del don como deber que lo despoja de su carácter festivo. Por otro lado, niega su carácter mercantil, impidiendo que el don se convierta en un sustituto del dinero o mercancía. 32 "La revolución de pensamiento que propone Hénaff consiste en desplazar el énfasis de la relación sobre el donante y el donatario y en buscar la clave del enigma en la mutualidad misma del intercambio entre protagonistas y llamar reconocimiento mutuo esta operación compartida." 33 La cosa dada y devuelta es "prenda del compromiso del donante con el don y sustituto de la confianza en la aparición del gesto de reciprocidad". El "presente" o don visibilizaría, haría presente, la relación de mutualidad como un reconocimiento, gestual y no hablado, que no se reconoce a sí mismo, pero se simboliza en el regalo. El valor del regalo reside en esta su carga simbólica. ${ }^{34}$

Pero ¿cómo sostener esto en nuestra sociedad mercantilizada al extremo? En ella cobran validez las figuras de "'el sofista rehabilitado', 'el comerciante legitimado', 'el autor retribuido', 'el terapeuta pagado"'. ${ }^{35}$ Más aún, la ganancia sin inhibiciones éticas hasta goza de un cierto prestigio. Con todo, ¿qué sería de nuestras sociedades sin los valores inconmensurables con cualquier precio? Ricoeur menciona al respecto la dignidad moral, la integridad del cuerpo humano y la experiencia de la belleza. Remite al juicio de gusto kantiano de la tercera Crítica, ya que tal juicio "carece de referente

${ }^{31}$ Cfr. Ricoeur, Paul, CR, p. 241; PR, p. 338.

${ }^{32}$ Ibidem, p. 242; 339.

${ }^{33} \mathrm{Cfr}$. Ibidem, pp. $242 \mathrm{~s}$.

${ }^{34}$ Cfr. Ibidem, p. 243.

${ }^{35} \mathrm{Cfr}$. Ibidem 
objetivo y que sólo se sostiene por su comunicabilidad". ${ }^{66}$ Cabe plantearse, entonces, cuáles son los gestos simbólicos que instituyen relaciones dereconocimiento, a despecho de los que las llevan a la ruina.

Ricoeur evoca el análisis ideal-típico de M. Weber en cuanto permite la intersección entre "precisión conceptual" y "ejemplificación empírica". ${ }^{37}$

Ahora la interlocutoraes Nathalie Zemon-Davis. EnsuobraThe Gift in sixteenth Century France, 38 la historiadora ve al "espíritu del don" -con sus "ideales para dar y recibir en diferentes medios sociales"- heredero de dos creencias profundamente arraigadas: una, la bíblica que marca la antecedencia del don divino como fundamento de la gratuidad también en las prácticas humanas: "Habéis recibido gratuitamente, dad gratuitamente" (Mt. 10, 8). La otra fuente es la ética de la liberalidad de la antigüedad clásica, a su vez recogida por los humanistas del Renacimiento: la mutualidad entre el donante y el donatario tiene su ejemplo en las Tres Gracias que se dan la mano. ${ }^{39}$ "Lastbutnotleast" estarían los vínculos de amistad y la generosidad entre vecinos. A su vez, el don cuenta con un tiempo propicio en las fiestas de cosecha, de año nuevo, fiestas litúrgico-patronales, las del ciclo vital del individuo y de la familia referidas al nacimiento, a los ritos de pasaje, al matrimonio, a la muerte. El don sigue operando en los legados y herencias. Sin embargo, todo esto coexiste con los intercambios mercantiles. Marcando las diferencias, ya entonces eran posibles los entrecruzamientos y préstamos recíprocos entre ambas formas de intercambio: la del don y la mercantil. ${ }^{40} \mathrm{Tal}$ es, por ejemplo, el caso del libro, en el que el autor dedica y el editor vende. Pero Ricoeur advierte también contra la posible infiltración de la corrupción, que -como sabemos- suele valerse de dones (fallidos, perversos).

Se pregunta Ricoeur:"¿Por qué dar? El compromiso en el don constituye el gesto que inicia todo el proceso. La generosidad del don suscita, no una restitución, que, en sentido propio, anularía el primer don, sino algo

${ }^{36}$ Ibidem

${ }^{37}$ Cfr. Ibidem

${ }^{38}$ Cfr. Zemon-Davis,Nathalie, Thegift in sixteenth Century France, University of Wisconsin Press, 2000.

${ }^{39}$ Cfr. Ricoeur, Paul, CR., p. 245.

${ }^{40} \mathrm{Cfr}$. Ibidem. 
como la respuesta a un ofrecimiento." ${ }^{41} \mathrm{El}$ primer don funciona como un modelo e incentivo para el segundo.Asimismo, en todo verdadero don está incluido el don de sí, que implica una demanda de reconocimiento, de ahí la importancia que cobra el recibir.

Retornando a la vía del ágape, éste implica un responder a un llamado surgido de la generosidad del don inicial. En la trilogía dar-recibir-devolver, Ricoeur pone el eje precisamente en el recibir, porque depende de la manera del recibir el modo cómo el donatario encara el devolver. En lenguas romances la gratitud equivale a reconocer. Ella "aligera el peso de la obligación de devolver y orienta a ésta hacia una reciprocidad igual a la que suscitó el don inicial" ${ }^{42}$ Es así como resalta nuevamente la inconmensurabilidad del don respecto de los procesos judiciales y respecto de las transacciones comerciales. Lo festivo, que le es propio, suele alimentarse con la energía del amor: ya sea erótico, familiar o societal.

Además, el don que palpita en el perdón, permite "abrir un espacio de esperanza en el horizonte de la política y del derecho en el plano postnacional e internacional, estos gestos ponen en marcha una onda de irradiación y de irrigación que, de modo secreto e indirecto, contribuye a la progresión de la historia hacia estados de paz." 43 Y con su habitual gesto hospitalario le da también la mano a la lucha por el reconocimiento en la medida en que supo alejar al deseo humano del apetito de poder y de la fascinación por la violencia.

\section{Consideraciones finales}

La temática del reconocimiento asume y subraya decididamentenuestra condición relacional. En sus sucesivos tratamientos porparte de Hegel, Honneth, Taylor y Ricoeur, echamos una mirada sobre las pérdidas y las ganancias que ofrece el camino recorrido. Por el impacto fuerte que la lucha a muerte por el reconocimiento en Hegel tuvo sobre la posteridad, opacó por completo la alternativa por él presentada por medio de la relación de

${ }^{41}$ Ibidem, p. 248.

42 Ibidem, p. 249.

${ }^{43}$ Ibidem, p. 250. 
reconocimiento mutuo que ya acontece entre hermano-hermana, tanto activa como pasivamente (reconozco y soy reconocido). Y se reconocen precisamente como libres individualidades. Además, es la Bildung un camino esencial para el despliegue del reconocimiento. Ni Honneth ni Ricoeur parecen advertirlo, y pierden precisamente un aspecto fundante de la eticidad (Sittlichkeit) no sólo clásica, sino también moderna. Honneth sí continúa la veta de la libertad y de la persona, que en su dignidad reclaman el reconocimiento. Sin embargo, no aparecen ya como decisivas en Ricoeur, o sólo de soslayo.

Se perfilan como ganancias los análisis de filosofía social de Honneth referidos a la resolución de los conflictos en la sociedad actual en base al Leitmotiv del reconocimiento mutuo. Sin embargo, como él mismo lo confiesa, lo hace a precio de una sociologización (Soziologisierung) de la problemática. ${ }^{44}$

Consideramos como gran contribución de Ricoeur, quien -recogiendo sus propios motivos ya madurados y las contribuciones de tantos otros en una sinfonía de la hospitalidad- nos propone nuevas posibilidades para construir paz a partir de la celebración del vínculo en el ágape. Con ello lo social mismo se ordena hacia una excedencia que apunta a otra dimensión. Instaura el don como llamado y la respuesta como gratitud, un incremento amoroso de ser que no se resigna frente a los restos de violencia albergados en el modelo de la lucha. Ante la pérdida de dignidad ética por la culpa y los conflictos que ella genera tanto Hege ${ }^{45}$ como Ricoeur apelan a la figura del perdón. Con todo, extrañamos en Ricoeur esta referencia al filósofo alemán. El perdón en Hegel cura las "heridas del espíritu" sin dejar cicatrices, conlleva la reconciliación de la comunidad espiritual, que ahora se hace capaz de recibir al Dios que aparece y circula en ella.

Pero cabe agregar que la originalidad de Ricoeur estriba precisamente en explorar (gracias a las posibilidades del francés y otras lenguas romances) las posibilidades del perdón en su parentesco con el don, como incansable y sanadora reapertura de la relación herida. El perdón no desecha la memoria, al contrario, la supone, pero la transforma: la "memoria inquieta" se convierte

${ }^{44}$ Cfr. Honneth, Axel, op. cit., nota 2, p. 109.

${ }^{45}$ Hegel Georg Wilhelm Friedrich, Phänomenologie des Geistes, pp. 471s. 
en "memoria feliz". ${ }^{46}$ En suma, dar-recibir-agradecer, esta circulación virtuosa y potenciada del reconocimiento, dispensa la más decisiva fuerza creadora y nutricia de vínculos.

Recibido: 5/2015; aceptado: 9/2015

${ }^{46}$ Ricoeur Paul, CR, p. 170. Siep le reconoce a Ricoeur esta excedencia respecto de la Anerkennung alemana. Cfr. op.cit., p. 21. 